\title{
Hermetic Package for Optical MEMS
}

\author{
F. Seigneur, Y. Fournier, T. Maeder, P. Ryser, J. Jacot \\ EPFL - IMT - LPM \\ Station 17 \\ CH - 1015 Lausanne \\ $+41216935945$ \\ frank.seigneur@a3.epfl.ch
}

\begin{abstract}
This article describes the design and fabrication of a hermetic LTCC package for an optical MEMS chip designed for space applications. The package must ensure electrical and optical connections, mechanical positioning, atmosphere control, and finally low thermally induced stress on the MEMS during final packaging operation.

The package consists of a 10-layer LTCC case with a recessed cavity for the MEMS chip, and glass lid (with antireflection coating and thin-film metallisation for soldering) for optical I/O. The chip is mechanically attached to the bottom of the cavity with a silicone adhesive, and electrically connected through gold wire bonds. The gold wire bonding pads are routed through the LTCC module to a MegArray BGA connector. Hermetic closure of the cavity is carried out by soldering the glass lid onto the case in a controlled atmosphere.

The two main difficulties involved in such a package are the high electrical connection density (400 connections) and low-temperature hermetic sealing. LTCC design rules for small-pitch lines, thick-and thin-film materials selection, screen-printing, lamination techniques and soldering methods are described in this article.
\end{abstract}

Keywords: LTCC, Hermetic Packaging, MOEMS, Interconnections, Solder Sealing.

\section{Introduction}

The field of Micro-Electro-Mechanical Systems (MEMS) enjoys rapid growth. Such devices often need special and precisely controlled conditions to operate correctly (temperature, inert gas, I/O interfaces, etc.). Often, development is mainly focused on manufacturing the MEMS device, and the final packaging is thus neglected. When the device to be encapsulated is at an advanced design stage, it is difficult to propose a convenient packaging method, which fulfils the required final specifications, together with manufacturing constraints. Long-term hermeticity and low sealing temperature are often difficult to achieve. Add the need of high electrical conductor density and optical interface, and the problem is even harder to solve. This paper describes a case study where the proposed packaging solution fulfils several external functions, together with constraints due to the sensitiveness to heat of the encapsulated MEMS.

The first part of this paper describes the device to be packaged and the functions to be fulfilled by the package. Then, design and manufacturing of the solution are described and discussed, focusing on critical operations. Finally, the methods used as well as potential improvements are discussed in the conclusion.

\section{Description of the problem}

The device to be packaged is an optical MEMS device designed for space telecommunication applications. It consists of 128 micro mirrors; each mirror requires 3 electrical connections for its electrostatic actuation. The MEMS chip is $35 \mathrm{~mm}$ long and $13 \mathrm{~mm}$ wide. Several critical functions need to be fulfilled by the package, including optical and electrical interfaces, atmosphere control and mechanical positioning. These functions are described below.

\section{Electrical connection}

The MEMS chip has to be electrically connected to a control electronics, by 386 bonding pads placed on two of its long sides. The bonding pads have a width of $120 \mu \mathrm{m}$ and a pitch of $150 \mu \mathrm{m}$. The space between two pads is $30 \mu \mathrm{m}$. 


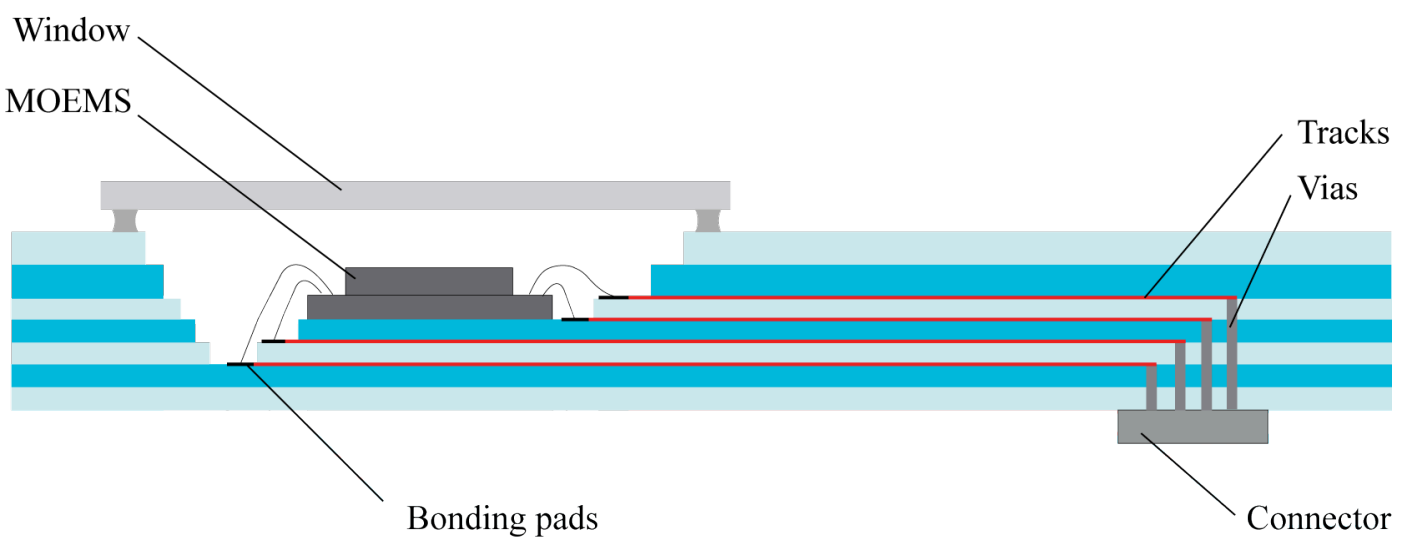

Figure 1 - Schematic view of the package.

\section{Optical interface}

The MEMS consists of an array of micro mirrors and needs a glass lid to allow an optical link to the outside. The glass is coated on both sides with an anti-reflective coating.

\section{Mechanical positioning}

The MEMS chip needs to be positioned precisely under an array of optical fibres.

\section{Atmosphere control}

The micro mirrors must operate in a dry atmosphere to ensure long-term device reliability and stability. Some damping is needed; they cannot reside in vacuum, but need a controlled pressure of an inert gas.

\section{Sensitivity to temperature}

The package has to ensure a low thermal budget on the MEMS during the sealing operation. The micro mirrors might deform when exposed to temperatures above $200^{\circ} \mathrm{C}$.

\section{Production volume and design
flexibility}

The volume to be produced is low (10 to 20 packages), but it must be possible to increase this volume up to 100 packages per year. The design has to be flexible, as changes can occur in both design of the MEMS and of the package during the development.

\section{Description of the solution}

Regarding the specifications described in the previous chapter, the following solution is proposed: the package is composed of two main parts. The base is made of LTCC (DuPont 951) [7], and a glass lid is soldered to the base (See Figure 1 and Figure 2).

The package is a $62 \times 62 \mathrm{~mm} \mathrm{10-layer}$ LTCC base with a recessed cavity for the MEMS chip. The chip is glued in the cavity. The chip is connected through wire bonds, the gold wire bonding pads are connected to a MegArray BGA connector [10] through electrical lines and vias.
The electrical lines, vias and bonding pads are screen-printed on the green tape. The bottom of the package consists of 5 tapes which receive the screen-printed tracks and bonding pads. These tapes are $114 \mu \mathrm{m}$ thick. The walls of the cavity are formed by 5 tapes which are $254 \mu \mathrm{m}$ thick.

The glass lid is soldered to the package, thus ensuring hermeticity. The glass receives an antireflective coating. A metallic frame is evaporated onto the glass, in order to allow wettability of the solder on the glass. To reduce the thermal impact on the MEMS device during encapsulation, all high-temperature operations are done before gluing the MEMS chip in the cavity. The final operation (bonding and soldering of the glass lid) are made at low temperature, reducing thermal impact on the MEMS device.

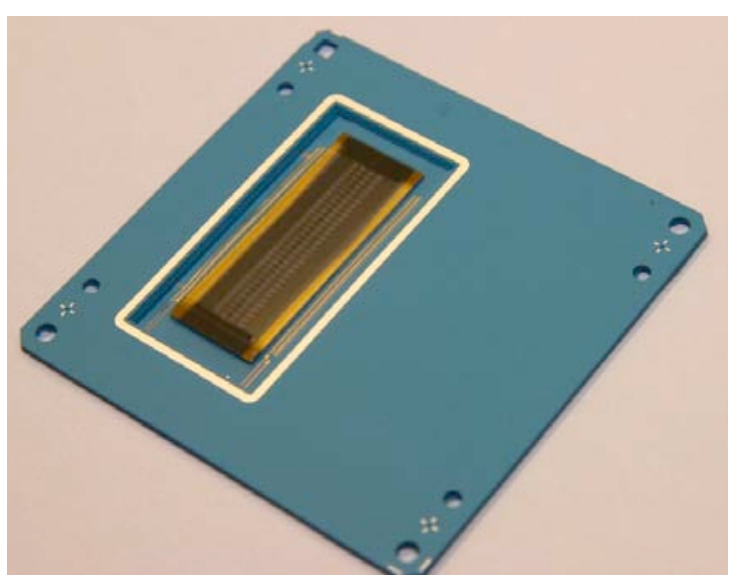

Figure 2 - Package with bonded chip, ready to be sealed.

\section{Design}

Although it might seem simple, as it implies only conductor lines between the chip and the connector, the routing needs several design steps. They are described below.

\section{Sketches}

The first step is to determine the critical pitches, this leads to a general routing. On the chip side, the bonding pads have a pitch of $150 \mu \mathrm{m}$. On 
the connector side, the pitch is 1'270 $\mu \mathrm{m}$. There is a factor of 10 between the chip and the connector; the package has to make the link between these two domains. Standard screen-printing allows a minimum pitch of $300 \mu \mathrm{m}$ (track width: $150 \mu \mathrm{m}$ ). It is convenient for the connector side, but a 2-layer solution is needed on the chip side. In order to simplify routing, a 4-layer solution was chosen which minimizes the number of vias (See Figure $1)$.

\section{Use of design software}

The second step is the design of the mechanical layout of the components, taking into account the previous remarks concerning the 4 layers needed. Routing is made in HYDE [8]. This software allows design of modules on several layers, and also takes shrinkage of LTCC into account. In our case, every track and via had to be drawn manually. The goal is to minimize the length of the tracks.

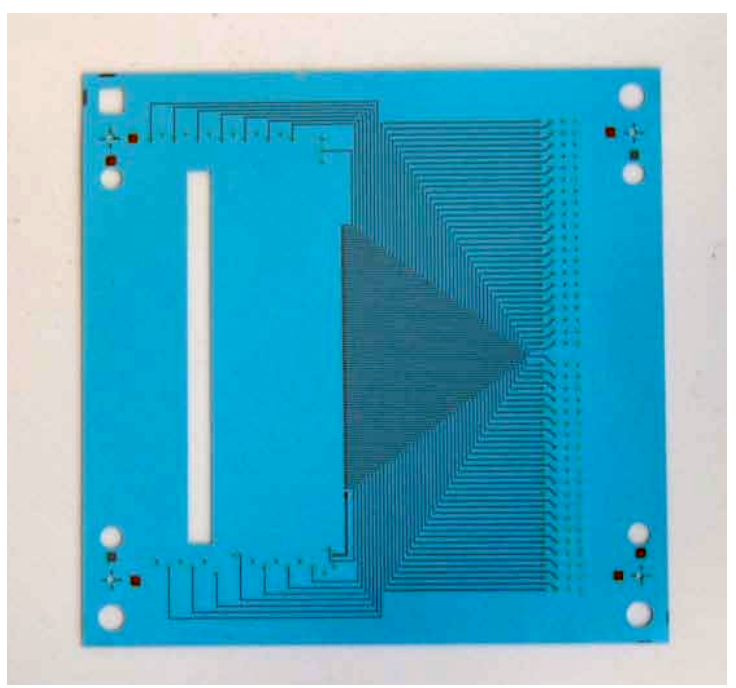

Figure 3 - Layout of tape 6.

\section{Design rules}

The fine pitch needed for screen-printing is hard to obtain. Some design rules need to be observed.

On the masks used for screen-printing, the mesh is orientated at $45^{\circ}$. The tracks cannot be parallel to the mesh, because of resolution issues. The best screen-printing resolution is obtained perpendicular to screen-printing direction, which means that the finest tracks must be parallel to the screen-printing direction.

The via diameter is equal to the thickness of the tape $(100 \mu \mathrm{m})$. To ensure a proper connexion even with small misalignments, the via pad diameter is $200 \mu \mathrm{m}$. The MegArray connector cannot be soldered directly over the vias. The soldering pads must be slightly offset from the vias.

\section{Paste choice}

Three different pastes fulfilling different functions are needed: bonding pads, tracks, vias. The main concern is about bonding as the equipment used allows only gold ball bonding. Therefore the paste for the bonding pads needs to be gold also (TC502 or DP5472). For the tracks, two different pastes were tested: DP6146 (Ag-Pd) and DP6145 (Ag). Two via fill pastes were also tested: DP6138 (Ag-Pd) and DP6141 (Ag).

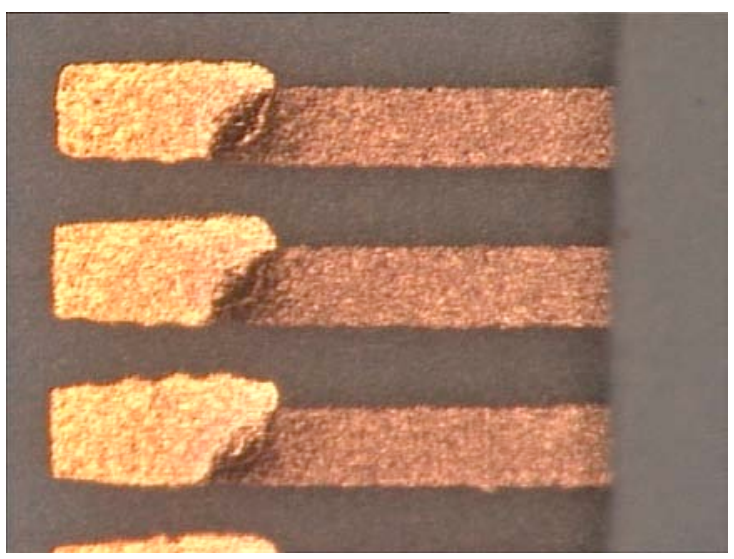

Figure 4 - Peeling between bonding pads (DP5472) and tracks (DP6146).

Concerning the via fill pastes, no significant difference is observed. The DP6141 is finally chosen. For the tracks, DP6146 exhibited better printability and compatibility during firing with the TC502 Au bonding pads, but implies more deformation during firing especially for high tracks density zones, where LTCC thickness is low.

For the bonding pads, peeling was observed between the bonding pad and the track when using DP5472 (See Figure 4). So finally, we chose TC502 for the bonding pads, DP6146 for the tracks, and DP6141 for the vias. The tested pastes are summarized in Table 1. The final choice is marked in bold.

Table 1 - Summary of pastes used for screenprinting.

\begin{tabular}{|l|l|l|}
\hline Type & Paste & Material \\
\hline Via fill (a) & DP6141 & Ag \\
\hline Via fill (b) & DP6138 & Ag-Pd \\
\hline Tracks (a) & DP6145 & Ag \\
\hline Tracks (b) & DP6146 & Ag-Pd \\
\hline Bonding pads (a) & TC502 & Au \\
\hline Bonding pads (b) & DP5472 & Au \\
\hline
\end{tabular}

\section{Manufacturing}

The package follows the standard LTCC manufacturing steps: pre-conditioning, laser cutting, screen-printing (vias, tracks, pads), lamination, firing and post-firing operations. Details are given on critical operations. 


\section{Screen-printing}

Each tape requires 3 screen-printing operations (via fill, tracks, bonding pads). The screen-printed paste needs to be dried at $120^{\circ} \mathrm{C}$ for 5 minutes before each new screen-printing operation. Due to the fine pitch, alignment is critical. The screen-printer used is a semi-automatic Aurel C900 equipped with pattern recognition and manual alignment. Alignment marks are used, but functional alignment is preferred when possible.

\section{Lamination}

The cavity is quite deep $(1 \mathrm{~mm})$, and the bottom has to be well laminated. Several changes to standard lamination procedures were needed to achieve good lamination quality. The top plate was fitted with a thick rubber sheet to achieve pseudoisostatic lamination of the bottom of the cavity (See Figure 5). The tapes forming the cavity are cut so that the walls are tapered to form an angle of $45^{\circ}$. The first tests were made using an unconstrained $12 \mathrm{~mm}$ thick rubber plate, positioned between the lamination press and the LTCC stack. A mylar sheet protects the LTCC from the rubber and the press. The result was not reproducible; deformation of the laminated LTCC stack occurred. When the rubber is compressed, its size in $\mathrm{x}$ and $\mathrm{y}$ increases, applying forces that deform the LTCC stack. To prevent this, the rubber plate was constrained in a circular aluminium tube (See Figure 6). The height of the rubber plate is slightly greater than the tube, allowing the rubber to get compressed by the lamination press. In this way, the deformation in $\mathrm{x}$ $\mathrm{y}$ is minimal. To further improve the homogeneity of the pressure, a second rubber is cut to the size of the cavity, and inserted in it before lamination.

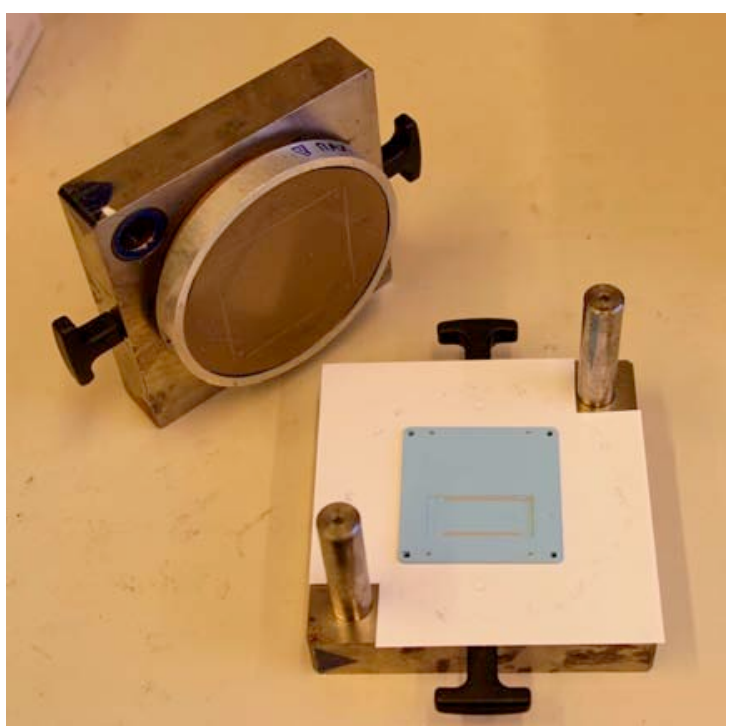

Figure 5 - View of LTCC stack before lamination. Note the constrained rubber plate.
The lamination pressure is 80 bars; most samples were laminated at ambient temperature $\left(25^{\circ} \mathrm{C}\right)$ and some were laminated at $55^{\circ} \mathrm{C}$, without a noticeable difference in the outcome. The mean shrinkage measured after firing is $14.1 \%$ and $13.7 \%$ in $\mathrm{x}$ and $\mathrm{y}$ respectively. This must be compared to $12.7 \%$ expected in $\mathrm{x}$ and $\mathrm{y}$, according to DuPont [7]. Previous work by Fournier et al. [3] showed an average shrinkage of $13.11 \%$.

The use of the rubber during lamination allows a good and quite reproductive lamination, using a much simpler process than isostatic lamination. However, there are some disadvantages: the top of the package is not perfectly flat as bumps corresponding to vias, tracks and alignment marks can be seen on the top of the package.

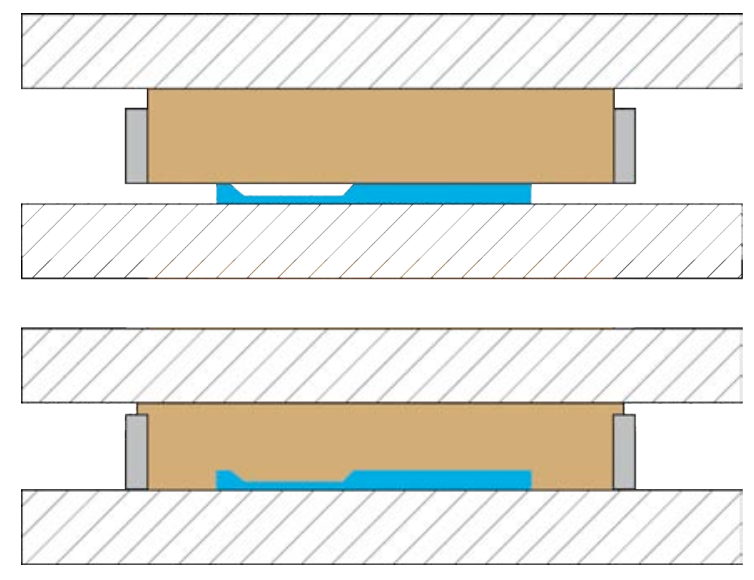

Figure 6 - Schematic view of the constrained rubber used for lamination.

Another issue concerning lamination is the high density of tracks. At some areas, the surface of the tracks is equal to the surface of the apparent LTCC. This leads to deformation due to different shrinkage coefficient between paste and LTCC. Also ensuring good lamination in this case is not trivial, because the lamination pressure and temperature must be limited to avoid excessive deformation of the package. To solve this, the surface of paste has to be reduced, and lamination has to be done with a rubber, to ensure good lamination of the LTCC between the tracks (See Figure 7). 


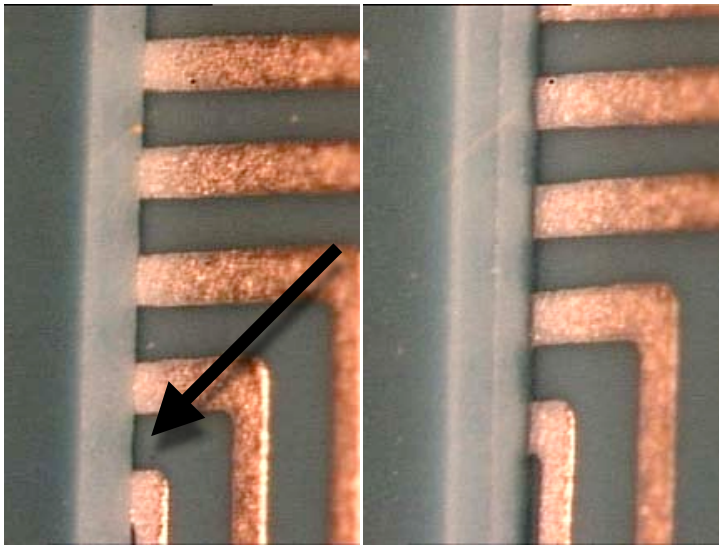

Figure 7 - Insufficient lamination (left); there is a gap between two LTCC layers; and good lamination (right).

Delamination was also observed during firing, in a high track density zone (See Figure 8). High track density implies low overlap of LTCC. Such zones must be avoided or a way must be found to allow stronger lamination without deformation. It appears that the firing profile plays a role in delamination, especially the temperature ramp during sintering (See Figure 9).

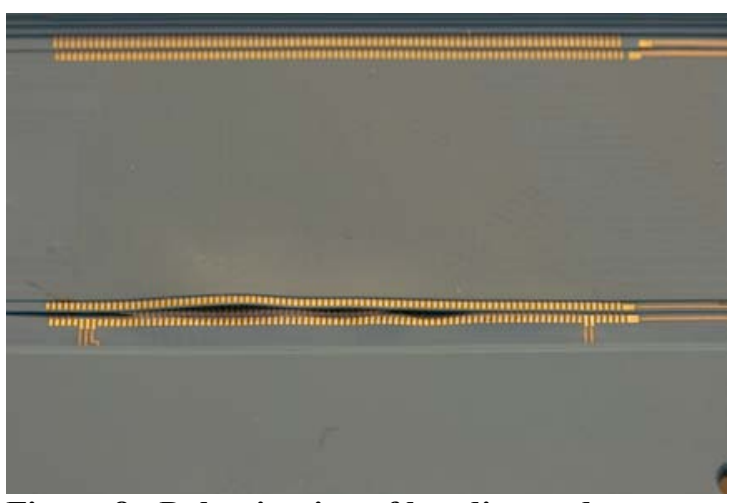

Figure 8 - Delamination of bonding pads.

Delamination occurred on some samples for ramps of $1.5 \mathrm{~K} / \mathrm{min}$. The best results were observed for ramps of $2.5 \mathrm{~K} / \mathrm{min}$. Storage time between the last screen-printing operation and lamination is also a factor that has an influence (up to two months storage time for samples showing delamination). This is due to the fact that the green tape dries out over time.

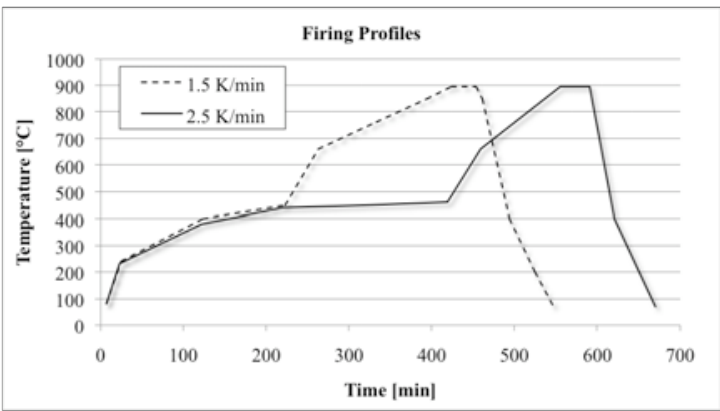

Figure 9 - Profiles used for firing.

\section{Post-operations}

The window metallization (DP6135D) is screen-printed and fired. Then an overglaze (ESL G481 or ESL G485-1, Pb-Free) is screenprinted as solder mask. Solder paste ( $\mathrm{Sn}-\mathrm{Cu}-\mathrm{Ag})$ is screen-printed for bump formation. Finally, the MegArray connector is placed manually onto the package and reflowed in an oven.

\section{Soldering of glass lid}

A metallization layer is needed in order to obtain wettability of the Sn-Bi eutectic solder on the glass lid. Previous work showed that screenprinted thick film worked well (ESL 590G - Ag), but the main drawback is the high required firing temperature $\left(500{ }^{\circ} \mathrm{C}\right)$ : the anti-reflection coating is damaged at temperatures above $300^{\circ} \mathrm{C}$. A low temperature solution is the evaporation of $\mathrm{Ti}$ $(20 \mathrm{~nm})-\mathrm{Pt}(200 \mathrm{~nm})-\mathrm{Au}(50 \mathrm{~nm})$ onto the glass. Thorough cleaning of the glass was found to be critical for achievement of good adhesion sputtering might be less sensitive in this regard.

The $\mathrm{Sn}-\mathrm{Bi}$ solder paste is manually dispensed on the metallic ring. It is first reflowed in an oven under vacuum in order to outgas the flux. The flux residue is then removed in an ultrasonic bath of TopKlean [9] during 15 minutes. Au ensures excellent solder wettability but must be kept thin in order to avoid the formation of brittle $\mathrm{Au}-\mathrm{Sn}$ intermetallics [11].

Glue is dispensed in the cavity, and the chip is manually aligned on it. The glue (EPOTEK $353 \mathrm{ND}$, epoxy) is cured for 1 hour at $80^{\circ} \mathrm{C}$. Finally, the chip is wire bonded.

After gluing of the chip in the cavity, all operations are done at low temperature $\left(150^{\circ} \mathrm{C}\right.$ or less), which ensures a minimal thermal impact on the chip. The soldering of the glass can be done in an oven, without risk of reflowing the MegArray connector, which was previously soldered with $\mathrm{Sn}$ $\mathrm{Cu}-\mathrm{Ag}$ (melting at ca. $220^{\circ} \mathrm{C}$ ). The main drawback of $\mathrm{Sn}-\mathrm{Bi}$ for soldering of the lid is sensitivity to creep at temperatures above $70^{\circ} \mathrm{C}$ [1] [2] [4]. Standard $\mathrm{Sn}-\mathrm{Pb}$ solder could be used instead, as its reflow temperature $\left(180^{\circ} \mathrm{C}\right)$ is still under that of Sn-Cu-Ag. A more elegant and efficient solution to the creep issue would be soldering of the lid with Sn-Cu-Ag also, using local laser heating.

When soldering the glass lid in an oven, the internal pressure due to temperature of the gas inside the package might break the liquid solder joint, leading to non-hermetic package. 


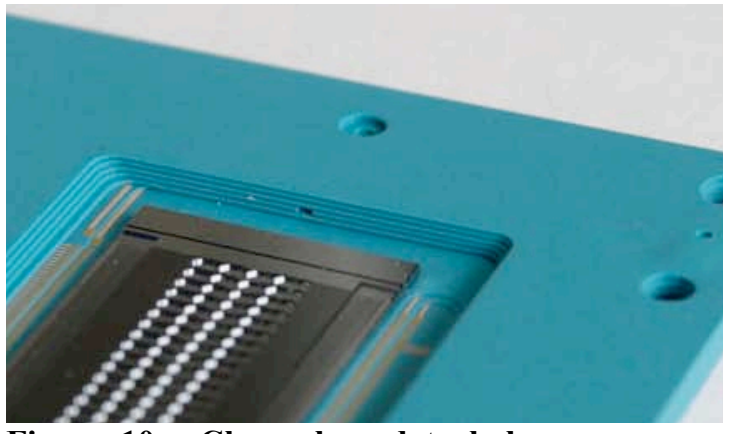

Figure 10 - Channel used to balance pressure during soldering.

To prevent this, a small channel is created in a wall of the package, which balances inside and outside pressures. The channel is cut in the tape (See Figure 10). To avoid collapsing during lamination, a small piece of cardboard is inserted in the channel. It is easily burnt during firing. After soldering of the glass, when the whole package is cold, the channel is sealed by a small soldered cap, which is heated locally.

\section{Potential costs}

An estimation of the development and manufacturing time is given in this chapter. The final cost for a batch of packages is composed of the design, the manufacturing and the material. The case study is given for a batch of 10 packages.

\section{Development and design}

Development time is related to complexity (i.e. number of connexions) of the MEMS chip to be encapsulated, but also to its requirements (sensitivity to temperature, protective atmosphere needed). One must also determine the mechanical properties of the package: size, mounting holes, layout of components and choice of the connector. In our case, one can estimate this time to four months, including drawing and layout in HYDE. This should be an iterative process with a close collaboration with the MEMS chip designer. Two persons are needed for this task, but their working rate is estimate at $50 \%$.

\section{Manufacturing}

This part consists in manufacturing of screens for screen-printing, laser cutting, screenprinting, stacking, lamination, firing, postoperations, gluing and bonding of the chip, electrical tests and sealing. The times for these tasks is summarized in Table 2.
Table 2 - Duration of manufacturing tasks

\begin{tabular}{|l|l|}
\hline Task & Duration \\
\hline Laser cutting & 4 days \\
\hline Screen-printing on green tape & 5 days \\
\hline Lamination and firing & 2 days \\
\hline Screen-printing & 2 days \\
\hline Gluing of chip and bonding & 5 days \\
\hline Electrical test and sealing & 5 days \\
\hline
\end{tabular}

\section{Material}

The material is a small part of the cost regarding to development and manufacturing. Nevertheless, they are summarized in the table below. This cost includes the LTCC base only, connector and glass lid are not included. The cost for one tape is $3 €$ including the paste. The total material cost for one package is estimated to be ca. $33 €$.

Table 3 - Cost of material for a batch of 10 packages.

\begin{tabular}{|l|l|l|}
\hline Tape & Qty & Cost (with paste), $€$ \\
\hline $1-5(114 \mu \mathrm{m})$ & 50 & 150 \\
\hline $6-9(254 \mu \mathrm{m})$ & 40 & 120 \\
\hline $10(114 \mu \mathrm{m})$ & 20 & 60 \\
\hline Totals & 110 & $330 €$ \\
\hline
\end{tabular}

\section{Conclusion and future work}

The proposed solution fulfils all the functions needed. It can easily be adapted to similar MEMS devices. The low development and manufacturing costs, together with design flexibility implies that this solution is to be considered for MEMS packaging.

Routing all 386 connections to one connector is quite challenging. The use of several smaller connectors would simplify the routing process, as they could be more easily distributed on the surface of the package.

The routing of the package should be symmetrical. The form factor of the package implies deformation and delaminations. It would be wiser to increase the thickness of the package, and reduce its surface, placing the connector directly under the chip.

Hermeticity has yet to be measured. Previous measurements were done on similar packages (LTCC and soldered glass lid) [5]. A gas sensitive LTCC module developed by Maeder et al. [6] could easily be integrated in such a package.

\section{References}

[1] Villain, J., T. Qasim, et al. "Struktur und Eigenschaften von Vapor-Phase umgeschnolzenen SnBi- und SnZn-Lötverbindungen an SMD- 
Bauelementen." IMAPS Deutschland Konferenz, München.

[2] Hua-F Glazer-J, "Lead-free solders for electronic assembly", Design \& Reliability of Solder Interconnects, proceedings, TMS symposium, 65-73, 1997.

[3] Y. Fournier, L. Bieri, T. Maeder, and P. Ryser. "Influence of lamination parameters on LTCC shrinkage under unconstrained sintering", 4th European Microelectronics and Packaging Symposium - IMAPS, pages 165-170, 2006.

[4] Wei, Y., C. L. Chow, et al. "Behavior of LeadFree Solder Under Thermomechanical Loading." Journal of Electronic Packaging 126: 367-373.

[5] F. Seigneur, T. Maeder, J. Jacot, "Laser Soldered Packaging Hermeticity Measurement Using Metallic Conductor Resistance", XXX International Conference of IMAPS Poland Chapter, Krakow, 24-27 September 2006.

[6] T. Maeder, Y. Fournier, F. Seigneur, P. Ryser, "LTCC active oxygen getter module for hermetic packaging applications", IMAPS/ACerS 4th International Conference and Exhibition on Ceramic Interconnect and Ceramic Microsystems Technologies, Munich, 2008.

[7] DuPont Green Tape 951 System, DuPont MCM, Microcircuit Materials, http://www2.dupont.com/MCM/en_US/

[8] HYDE, Durst CAD/Consulting GmbH, Holzgerlingen, Germany.

[9] TopKlean, Avantec Performance Chemicals, 94683 Vincennes CEDEX, France.

[10] MegArray Connector 84740-102LF, FCI, http://www.fciconnect.com

[11] Kalenik-J Kisiel-R Mlozniak-A Szczepanski$\mathrm{Z}$, "Some properties of $\mathrm{SnAg}$ and $\mathrm{SnAgCu}$ solder joints on printed circuit boards", Proceedings, XXVIII International Conference of IMAPS Poland Chapter, Wroclaw, 277-280, 2004. 